\title{
Effects of endoscopy-related procedure time on musculoskeletal disorders in Japanese endoscopists: a cross-sectional study
}

\section{(ㄷ)(1) $(-)$}

Authors

Ippei Matsuzaki ${ }^{1}$, Takeshi Ebara², Mafu Tsunemi ${ }^{3}$, Yoshifumi Hatta², Kojiro Yamamoto², Akemi Baba ${ }^{4}$, Masashi Hattori $^{1}$, Masanao Nakamura ${ }^{5}$, Mitsuhiro Fujishiro ${ }^{5}$

Institutions

1 Department of Gastroenterology, Yamashita Hospital, Ichinomiya, Japan

2 Department of Occupational and Environmental Health, Nagoya City University Graduate School of Medical Sciences and Medical School, Nagoya, Japan

3 Department of Nursing, Yamashita Hospital, Ichinomiya, Japan

4 Department of Clinical laboratory, Yamashita Hospital, Ichinomiya, Japan

5 Department of Gastroenterology and Hepatology, Nagoya University Graduate School of Medicine, Nagoya, Japan

submitted 29.9.2020

accepted after revision 30.11.2020

\section{Bibliography}

Endosc Int Open 2021; 09: E674-E683

DOI 10.1055/a-1352-3850

ISSN 2364-3722

(C) 2021. The Author(s).

This is an open access article published by Thieme under the terms of the Creative Commons Attribution-NonDerivative-NonCommercial License, permitting copying and reproduction so long as the original work is given appropriate credit. Contents may not be used for commercial purposes, or adapted, remixed, transformed or built upon. (https://creativecommons.org/licenses/by-nc-nd/4.0/)

Georg Thieme Verlag KG, Rüdigerstraße 14,

70469 Stuttgart, Germany

\section{Corresponding author}

Takeshi Ebara, PhD, CPE, Department of Occupational and Environmental Health, Nagoya City University, Graduate School of Medical Sciences, Mizuho-ku, Nagoya, 4678601, Japan

Fax: +81-52-859-1228

ebara@med.nagoya-cu.ac.jp

\section{ABSTRACT}

Background and study aims There has been little evidence assessing the prevalence of musculoskeletal disorders (MSDs) among endoscopists performing recent diagnostic and therapeutic endoscopic procedures requiring prolonged procedural times. We evaluated the prevalence and identified the risk factors for developing MSDs, focusing on procedural time.

Methods An electronic survey of endoscopists $(n=213)$ employed at the Nagoya University Hospital and its affiliated hospitals was developed by a multidisciplinary group. Results Of the 110 endoscopists (51.6\%) who responded to the survey, eighty-seven endoscopists $(79.1 \%)$ had experienced endoscopy-related MSDs during the previous 1 year, and 49 endoscopists (44.5\%) had experienced these MSDs during the previous week. Nineteen endoscopists (17.3\%) reported absence from work due to severe MSDs. The most frequent sites of MSDs were neck, low back, and shoulders. Logistic regression analyses showed that longer upper endoscopic submucosal dissection ESD, (odds ratio: 5.7; 95\% Cl: 1.3-25.0), lower ESD (odds ratio 4.9; $95 \% \mathrm{Cl}$ : 1.1-22.0), and lower gastrointestinal treatment (odds ratio: 5.6 ; $95 \% \mathrm{Cl}: 2.3-13.3)$ were significantly associated with the development of MSDs in the low back area. Moreover, longer lower ESD (odds ratio: 5.0; 95\% Cl: 1.2-20.2) was a risk factor for symptoms in the left shoulder.

Conclusion This study suggests a correlation between the volume of therapeutic endoscopic procedures including ESD and the risk of MSDs mainly low back area and left shoulder. Managing monthly total endoscopic time, in light of organizational ergonomics, could contribute to minimizing such risks of endoscopy-related MSDs. 


\section{Introduction}

Frequent, repetitive maneuvers and awkward body positions may lead to musculoskeletal disorders (MSDs) [1]. Work-related MSDs have been reported in individuals with various occupations, such as ultrasonographers, and surgeons [2,3]. Endoscopists are also at high risk of developing MSDs. Several studies have reported the prevalence of endoscopy-related MSDs to range from $20 \%$ to $89 \%$. Common sites of pain and injuries are the thumbs, wrists, neck, and low back [4-12]. The development of MSDs in endoscopists translates to cost for the hospital and society. Furthermore, this leads to longer waiting times for patients and poor endoscopic performance. Risk factors of endoscopy-related MSDs include sex, seniority, improper position during the procedure, and endoscopy-specific maneuvers (e.g., torqueing) during colonoscopy [4-12]. Other reported risk factors are high procedure volume and prolonged duration of endoscopy, which leads to overuse injury due to repetitive movements $[4,5,9,13]$.

Diagnostic and therapeutic endoscopic procedures requiring prolonged procedural times, such as endoscopic submucosal dissection (ESD), enteroscopy, and endoscopic ultrasound (EUS), are being performed with increased frequency in clinical practice. Due to the duration of these procedures, this may predispose endoscopists to higher rates of MSDs than previously reported. However, there have been a few studies proving the correlation between endoscopy volume and risk of MSD development including these recent developed procedures $[9,12$, 14].

The aim of this study was to evaluate the prevalence of MSDs and identify the risk factors for developing MSDs, focusing on procedure time among endoscopists in Japan.

\section{Methods}

\section{Survey sampling}

We conducted an electronic cross-sectional survey of endoscopists employed at the Nagoya University Hospital and its affiliated hospitals with registered e-mail addresses $(n=55)$ between October 2019 and November 2019. Members who were currently performing endoscopy were eligible to participate. The institutional review board of Yamashita Hospital (YEC1904) approved the study prior to the dissemination of the survey.

\section{Survey instrument}

The survey was performed using a 50-item, self-administered, electronic questionnaire developed by a multidisciplinary group consisting of two endoscopists (I.M., M.F.), one endoscopy nurse (M.T.), one endoscopy technician (A.B.) and two ergonomists (T.E., K.Y.).

The items included in the survey measured the characteristics of endoscopists, workload parameters, and experience during and after the participants experienced an injury. The characteristics of endoscopists included age, sex, height, weight, hand dominance, main avocational activities, and practice setting. Workload parameters included the number of years in practice, hours and number and/or type of endoscopies con-
Requested the responsible endoscopist working at 55 hospitals in Aichi prefecture, Japan

25 hospitals agreed on participation (47.3\%)

Inclusion criteria

- Endoscopists currently working full time

Exclusion criteria

- Endoscopists on temporary leave due to maternity leave and childcare leave

Persons matched the eligibility criteria $(n=213)$

110 (51.6\%) were responded

Handling missing data

- Multiple imputation was applied prior to the calculations to impute the missing values

- The following variables were used as predictors and outcomes in the models: demographic variables such as sex, height, weight and factors used in the logistic regression models.

Inclusion in analyses $(n=110)$

- Fig. 1 Participant selection flowchart.

ducted per month, length of time spent performing procedures, and working posture. Injury experiences included the location of pain or injury and the effects of the injury on the occupation of the participants.

The Standardized Nordic Musculoskeletal Questionnaire was used to assess the prevalence of MSDs experienced pain, numbness, and discomfort on local body parts as primary outcomes [15]. The duration of the survey was approximately 15 minutes.

\section{Survey data collection}

- Fig. 1 shows the flowchart of selection of participants. We sent an invitation letter to 55 directors of hospitals, four academic centers, 14 large hospitals ( $\geq 5$ specialists performing endoscopy), and 37 small hospitals in Japan. Of these, 25 hospitals, one academic center, nine large hospitals, and 15 small hospitals agreed to participate in this study. The director of each participating institution requested endoscopists working at each hospital to voluntarily participate in the online survey via e-mail. The e-mail described study objectives, identified the research team and provided a direct link to the online survey (prepared by Google form), which was valid for a period of two weeks. We did not send a second reminder email, even if the participants did not respond within 2 weeks after the initial email. Informed consent was obtained on the website, which 


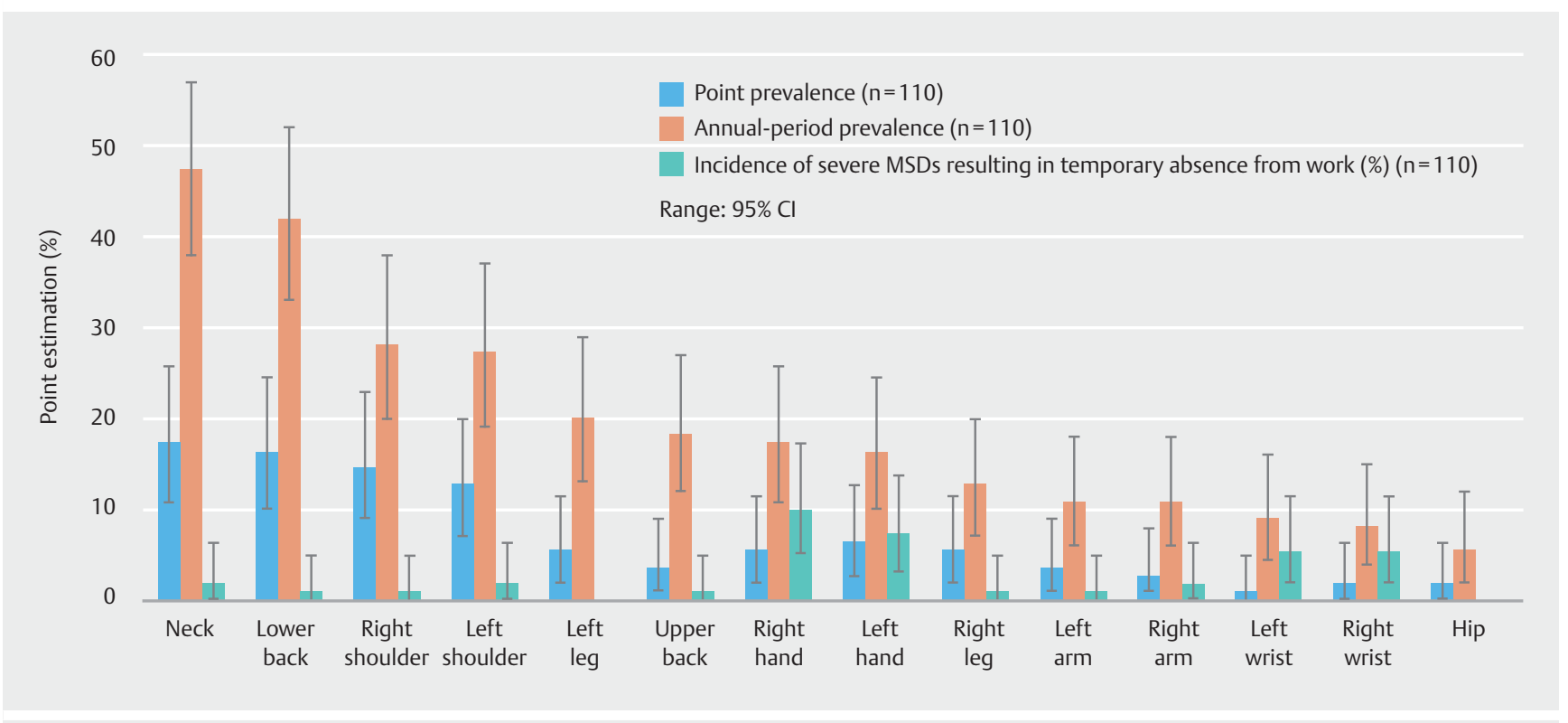

-Fig. 2 Point/annual-period prevalence of MSDs and incidence of severe MSDs on local body site.

was required prior to filling out the questionnaire and served as respondent's agreement to participate in the study.

The Yamashita Hospital Research Group (I.M., M.T.) administered the survey and data were directly stored into a database. All data remained anonymous to prevent response bias. Responders were not remunerated for their participation.

\section{Statistical analysis}

Demographic characteristics of the respondents were summarized using the mean with standard deviation for continuous variables and count with proportion for discrete ones. Welch's $t$ test adjusting degrees of freedom for unequal variances was adopted to estimate the significant difference of continuous data between two groups. Fisher's exact test was used to compare the distributions between nominal data. For estimating the point/annual prevalence of MSDs as primary outcomes, its $95 \%$ confidence interval $(\mathrm{Cl})$ were calculated.

We adopted a binary logistic regression model to extract potential risk factors on each endoscopy-related procedure time affecting MSDs of neck, shoulders, and low back. Each procedure time was reclassified as categorical variables using quartile, or was distinguished them based on features of multimodal distribution. Our data had quite low missing values of only one or two cases in each estimation model though, we dared to apply the multiple imputation method to the cases to reduce the effect of potential biases in such a case of small sample size [16, 17].

Pooled adjusted odds ratios (ORs) were calculated after applying the multiple imputation to handle missing data. Such a multiple imputations method is widely recommended for correction of potential biases attributed to non-respondents and is considered more efficient in most settings. Adjustments against confounders were made for the following variables: sex, age, certified fellow of endoscopy, seniority (yrs), working hours (h/wk), and sedentary time (h/d).

All statistical analyses were carried out with statistical software package SPSS 24.0 (SPSS, Chicago, Illinois, United States).

\section{Results}

\section{Point/annual-period prevalence of MSDs and incidence of severe MSDs among endoscopists}

Of the 213 invited endoscopists in the 25 hospitals, 110 (51.6\%) responded. The majority of endoscopists $(n=87 ; 79.1 \%$ ) had experienced an endoscopy related-MSDs in at least one anatomic location during the previous 1 year (annual-period prevalence of MSD). The majority of endoscopists ( $n=49 ; 44.5 \%$ ) also had experienced an endoscopy related-MSDs in at least one anatomic location during the previous week (point-period prevalence of MSD). Nineteen endoscopists (17.3\%) reported absence from work as a result of injury (severe MSD). In addition, 11 endoscopists (10.0\%) consulted doctors.

- Fig. 2 shows the point/annual-period prevalence of MSDs and incidence of severe MSDs on local body site. The most frequent sites of annual-period prevalence of endoscopy relatedMSD were the neck $(n=52 ; 47.3 \%)$, low back $(n=46 ; 41.8 \%)$, right shoulder ( $n=31 ; 28.2 \%)$, and left shoulder $(n=30 ; 27.3 \%)$. For the point-period prevalence, the most frequent sites were the neck ( $n=19 ; 17.3 \%)$, low back $(n=18 ; 16.4 \%)$, right shoulder $(n=16 ; 14.5 \%)$, and left shoulder $(n=14 ; 12.7 \%)$. In contrast, the most frequent sites of severe MSDs resulting in temporary absence from work were the right hand $(n=11 ; 10.0 \%)$, left hand $(n=8 ; 7.3 \%)$, left wrist $(n=6 ; 5.5 \%)$, and right wrist $(n=$ $6 ; 5.5 \%)$. 


\section{Risk factors for developing endoscopy related MSDs}

To investigate the risk factors for developing endoscopy-related MSDs, we focused on local body sites with high prevalence: neck, shoulders, and lower back. - Table 1 shows demographic characteristics of endoscopists between those who reported endoscopy-related MSDs currently perceived on the neck, shoulders, or low back ( $n=37 ; 33.6 \%)$ and those who had no reported endoscopy-related MSDs. The characteristics of respondents without MSDs were similar to ones with MSDs except in sex $(P=0.06)$ and body mass index (BMI, $P=0.05)$. On average, they spend $41.6 \%$ of their working hours engaged in endoscopic work. Comparing endoscopists who reported MSDs and those who did not, there was no differences in procedure duration in either group when performing the same procedure.

Working posture under each type of procedure is shown in - Table 2. More than $90 \%$ of the endoscopists selected the standing position during the endoscopic examination and procedure.

Results of the multivariate analysis regarding potential predictors of endoscopy-related MSDs on the neck, shoulders, and low back area are shown in $>$ Table 3. Longer upper ESD (total time: $\geq 181 \mathrm{~min} /$ month, OR: $5.7 ; 95 \% \mathrm{Cl}: 1.3-25.0$ ) and lower ESD (total time: 1-90 min/month, OR 4.9; $95 \% \mathrm{Cl}: 1.1-$ 22.0), and lower gastrointestinal treatment (total time: $\geq 526$ min/month, OR: 5.6; $95 \% \mathrm{Cl}: 2.3-13.3)$ were significantly associated risk factors for symptoms in the low back area. Moreover, lower ESD was a significantly associated risk factor for symptoms in the left shoulder (total time: $91-180 \mathrm{~min} / \mathrm{month}, \mathrm{OR}$ : 5.0; $95 \% \mathrm{Cl}: 1.2-20.2)$.

\section{Discussion}

\section{Features of endoscopy-related MSDs}

This study revealed a high prevalence of endoscopy-related MSDs (annual-period: $79.1 \%$, point-period: $44.5 \%$ ), including $17.3 \%$ of respondents reporting absence from work. This study also corroborates the finding of other studies in correlating a higher number of procedural hours with the development of MSDs. The most frequent sites for the annual/point-period prevalence of endoscopy-related MSDs in our survey were the neck, low back, right shoulder, and left shoulder. On the other hand, endoscopy related-MSDs were not frequently observed in the hands and wrists. In contrast, a high prevalence of MSD on these sites was reported in several previous reports [4-12]. These sites of MSDs may be influenced by the definition of MSD prevalence, technical skill for operating the endoscope, and hand size.

\section{Definition of MSD prevalence}

Such discrepancy can be found in the definition of outcomes between studies. Some studies distinguished endoscopy-related MSDs and non-endoscopy-related MSDs [9, 12]. The current study did not determine the work-relatedness of MSDs to endoscopy because we thought respondents could not definitively report this association. However, the current study examined three outcomes: annual prevalence, point prevalence, and incidence of severe MSDs. These differences in definitions may influence the results related to the prevalence of MSDs in these sites. In our study, severe MSDs were often observed in the hands and wrists.

\section{Technical skill for operating the endoscope}

Villa et al. also reported that MSDs occurred mostly during the first 3 years of fellowship $(85 \% ; P<0.001)$ in the United States, suggesting that the most common sites of MSDs were the right wrist (53\%), left thumb (42\%), back (27\%), and neck (22\%). Only $26 \%$ of those with endoscopy-related MSDs had received training in ergonomics versus $45 \%$ of those without injuries ( $P$ $=0.012$ ) [11]. Similarly, some studies reported differences in the sites of endoscopy-related MSDs between beginners and experienced endoscopists $[6,10]$.

Endoscopists have to perform endoscopy-specific maneuvers, such as adjusting the tip of angulation controls of the endoscope using the left thumb, and strongly torqueing using the right wrist in colonoscopy $[5,13]$. Furthermore, the activity of the left-wrist extensors, left-thumb extensors, and rightwrist extensors exceeded the hand activity level action limit during routine colonoscopy established by the American Conference of Industrial Hygienists [13]. Trainees use markedly more clockwise torque using the right wrist compared with experts during the colonoscopy technique [18]. In contrast, in a recent European study, the prevalence of hand numbness and wrist pain was significantly higher in consultants than fellows. The sample size of fellows $(n=38)$ was smaller in comparison with that of consultants $(n=133)$ in the previous study, and this difference may be responsible for the observed discrepancy in the results [12].

In the current study, the seniority of responders was relatively high (> 4 years, $94 \%$ ) and $83 \%$ of responders had completed a specialty (i.e., certified fellow of endoscopy). These characteristics of responders may have influenced the low frequency of the annual/point-period prevalence of MSD in the hands and wrists. Trainees may have to learn the neutral thumbs grip position, pinkie maneuver, and methods for the manipulation of the endoscope to avoid hand and wrist injuries [19].

\section{Hand size}

Hand size is a significant determinant of difficulty in using surgical instruments and affects the control of an endoscope [5, 20]. In a survey conducted in the USA, involving 1,295 gastroenterology fellows, 93 responders $(41.0 \%)$ considered the size of their hands to be inadequate for handling a standard endoscope. Of the 38 responders with a glove size $\leq 6.5$ inches, 37 (97.4\%) were female, expressing a wish to use endoscopes with smaller handles, if available [21]. Previous reports and the current study on the prevalence of endoscopy-related MSDs did not evaluate the hand size of endoscopists [4-11]. A recent European study evaluated the relationship of glove size to MSDs: small (32.8\%), medium (47.1\%), large (17.6\%), and extra-large (2.5\%) $(P=0.734)$ [12]. There is a possibility that the hand size of endoscopists influences the sites of MSD prevalence. Several studies have reported that the prevalence of endoscopy-related MSD leading to absence from work ranges from $3 \%$ to $18.5 \%$ 
- Table 1 Demographic characteristics of respondents.

\section{Variables}

Overall $(n=110)$

Age (\%)

- Less than 29

- 30 to 39

- 40 to 49

- 50 to 59

- 60 or older

$\operatorname{Sex}(\%)$

- Male

- Female

BMI $\left(\mathrm{kg} / \mathrm{m}^{2}\right.$, mean $\left.\pm \mathrm{SD}\right)$

Seniority (years, \%)

- Less than 3

- 4 to 6

- 7 to 9

- 10 to 15

. $\leq 16$

- Missing

$1(0.9)$

$61(55.5)$

$21(19.1)$

$23(20.9)$

4 (3.6)

$98(89.1)$

$12(10.9)$

23.1 (2.9)

$6(5.5)$

$18(16.4)$

$20(18.2)$

27 (24.5)

38 (34.5)

$1(0.9)$

Dominant hand

- Right

- Left

Specialty (certified fellow of endoscopy)

- No

- Yes

Practice setting (\%)

- Academic center

- Large hospital

- Small hospital

Working hours (h/wk, mean \pm SD)

Endoscopic work (\%, mean \pm SD)

Type of procedure (min/month)

- Upper Gl endoscopy

- Lower Gl endoscopy

- Upper ESD

- Upper GI treatment

- Lower ESD

- Lower Gl treatment

- Enteroscopy

- ERCP

105 (95.5)

5 (4.5)

27 (24.5)

83 (75.5)

34 (30.9)

35 (31.8)

41 (37.3)

$54.8(11.4)$

$41.6(21.9)$

$380.1(229.0)$

565.5 (476.9)

102.1 (128.5)

$53.2(71.2)$

$70.4(102.6)$

$448.0(465.4)$

$93.6(141.3)$

206.9 (305.1)
Those who have any endoscopy-related MSDs currently perceived on neck, shoulders or low back

No $(n=73)$

Yes $(n=37)$

$0(0)$

$1(1.4)$

$21(56.8)$

$14(19.2)$

7 (18.9)

$16(21.9)$

$7(18.9)$

$2(2.7)$

2 (3.6)

62 (84.9)

$11(15.1)$

$22.7 \pm 2.5$

$36(97.3)$

0.06

$1(2.7)$

$23.8 \pm 3.3$

0.05

0.93

\begin{tabular}{l|l}
\hline $4(5.6)$ & $2(5.4)$ \\
\hline
\end{tabular}

0.97

$13(18.1)$

$5(13.5)$

$12(16.7)$

$8(21.6)$

18 (25.0)

9 (24.3)

25 (34.7)

$13(35.1)$

70 (95.9)

35 (94.6)

1.00

$3(4.1)$

2 (5.4)

$19(26.0)$

$8(21.6)$

0.65

$54(74.0)$

$29(78.4)$

25 (34.2)

9 (24.3)

0.36

20 (27.4)

$28(38.4)$

$55.3(10.4)$

$40.6(22.3)$

15 (40.5)

$13(35.1)$

$53.7(13.3)$

0.51

$43.5(21.1)$

0.51

384.8 (225.8)

$605.5(537.1)$

370.9 (238.0)

0.77

84.7 (93.6)

$49.3(66.3)$

$65.6(95.6)$

$436.6(492.0)$

$82.0(140.3)$

$217.6(356.6)$

\begin{tabular}{|l|l|}
\hline $486.5(319.4)$ & 0.15 \\
\hline
\end{tabular}

$136.6(175.1)$

0.10

$60.8(80.3)$

0.46

$79.9(115.9)$

0.52

470.3 (413.3)

0.71

$116.4(142.5)$

0.23

185.7 (163.8)

0.52 
$>$ Table 1 (Continuation)

Variables

\begin{tabular}{|l|c|c|c|c|}
\hline & Overall $(\mathbf{n}=\mathbf{1 1 0})$ & No $(\mathbf{n}=\mathbf{7 3})$ & Yes $(\mathbf{n = 3 7 )}$ & $\mathbf{P}$ \\
\hline - EUS & $99.7(118.6)$ & $101.5(126.2)$ & $96.1(103.4)$ & 0.81 \\
\hline Sedentary time (h/d, mean \pm SD) & $3.8(2.7)$ & $3.7(2.5)$ & $3.9(3.0)$ & 0.80 \\
\hline
\end{tabular}

MSD, musculoskeletal disorders; BMI, body mass index; GI, gastrointestinal; ESD, endoscopic submucosal dissection; ERCP, Endoscopic Retrograde Cholangiopancreatography; EUS, endoscopic ultrasound; Fisher's exact test or Welch's $t$ tests were used to test for differences between groups.

- Table 2 Endoscopy-related work and working postures.

\begin{tabular}{|c|c|c|c|c|}
\hline & \multirow[b]{2}{*}{$\mathbf{n}$} & \multicolumn{3}{|l|}{$\%$} \\
\hline & & Standing & Sitting & sit-stand \\
\hline Upper GI endoscopy & 110 & $108(98.2)$ & $2(1.8)$ & - \\
\hline Lower Gl endoscopy & 106 & $101(95.3)$ & $2(1.9)$ & $3(2.8)$ \\
\hline Upper ESD & 67 & $64(95.5)$ & $3(4.5)$ & - \\
\hline Upper GI treatment & 81 & $81(100.0)$ & - & - \\
\hline Lower ESD & 52 & $47(90.4)$ & $1(1.9)$ & $4(7.7)$ \\
\hline Lower GI treatment & 105 & $100(95.2)$ & $2(1.9)$ & $3(2.9)$ \\
\hline Enteroscopy & 38 & $38(100.0)$ & - & - \\
\hline ERCP & 75 & $75(100.0)$ & - & - \\
\hline EUS & 73 & $73(100.0)$ & - & - \\
\hline
\end{tabular}

$[6-9,11,12]$. Notably, in the current study, $17.3 \%$ of endoscopists had to be absent from work due to MSDs, and the most frequent sites of severe MSDs resulting in temporary absence from work were the hands and wrists. Regardless of the MSD prevalence, we should focus on hand and wrist injuries. Future surveys should investigate the hand size of endoscopists and manufacturers should develop safer and more user-friendly endoscopes [8, 22-24].

\section{Endoscopy-related procedure time affecting MSDs}

The risk of MSD development appears to be influenced by the increased volume of endoscopies. Higher procedure volume (>20 cases/week, $P<0.001)$, a greater number of hours per week spent performing endoscopy ( $>16$ hours/week, $P<0.001$ ), and the total number of years performing endoscopy ( $>16$ years, $P=0.004$, ) are associated with a higher rate of endoscopy-related MSDs [9]. Similarly, cardiac ultrasonographers experienced injuries at a significantly higher rate when the number of examinations performed per month exceeded 100 [2]. Workloadassociated factors, such as the number of procedures or duration of work, were also related to the prevalence of MSDs in other occupations [25]. Alternatively, the ASGE survey did not show a meaningful relationship between the time spent performing several endoscopic procedures and the prevalence of endoscopy-related MSDs [9]. The endoscopic examination and procedure exhibit variability in procedure time, posture, and specifications of the endoscope in comparison with ultrasonography. Therefore, we confirmed the risk of endoscopy-related procedure time affecting MSDs through a multivariate analysis of endoscopy-related MSD in each site (i.e., neck, shoulders, and low back).

\section{Neck MSDs}

The endoscopy-related procedure time was not a significant risk factor for developing symptoms in the neck. Generally, the importance of proper setup of the monitor that allows for neutral postures has been established in surgery. In the horizontal plane, the monitor should be placed straight in front of the operator to avoid axial rotation of the spine. In the sagittal plane, the monitor should be positioned lower than the eye level to avoid neck extension [3,26]. To date, verification experiments regarding the appropriate position of the monitor in an endoscopic unit have not been performed. According to ergonomics, several reports suggested the appropriate position to prevent neck strain [22-24,27-29]. It is, however, out of the scope of this study. Hence, our survey could not deal with information regarding the monitor setting in each endoscopic pro- 
- Table 3 Relative risk of endoscopy-related procedure time affecting local body site on musculoskeletal pain.

\begin{tabular}{|c|c|c|c|c|c|c|c|c|}
\hline \multirow{2}{*}{$\begin{array}{l}\text { Total time } \\
\text { engaged in } \\
\text { (min/month) }\end{array}$} & \multicolumn{8}{|c|}{ Pooled adjusted OR (95\%CI) } \\
\hline & Neck & $P$ & Right shoulder & $P$ & Left shoulder & $P$ & Low back & $P$ \\
\hline \multicolumn{9}{|c|}{ Upper GI endoscopy } \\
\hline - $0-150$ & 1 & & 1 & & 1 & & 1 & \\
\hline - $150-350$ & $2.7(0.4-16.8)$ & 0.29 & $2.3(0.4-14.1)$ & 0.38 & $1.7(0.2-13.8)$ & 0.61 & $0.8(0.1-3.9)$ & 0.73 \\
\hline - $351-550$ & $3.3(0.5-22.7)$ & 0.23 & $0.3(0.0-4.4)$ & 0.41 & $2.4(0.3-19.9)$ & 0.42 & $0.2(0.0-1.3)$ & 0.09 \\
\hline - $551 \leq$ & $1.8(0.2-16.5)$ & 0.59 & $3.2(0.4-28.7)$ & 0.29 & $3.9(0.3-43.4)$ & 0.27 & $0.4(0.1-3.1)$ & 0.40 \\
\hline \multicolumn{9}{|c|}{ Lower Gl endoscopy } \\
\hline - $0-250$ & 1 & & 1 & & 1 & & 1 & \\
\hline - $251-750$ & $1.6(0.5-5.6)$ & 0.43 & $2.5(0.6-10.1)$ & 0.19 & $1.3(0.3-5.2)$ & 0.70 & $0.8(0.4-1.5)$ & 0.70 \\
\hline . $751 \leq$ & $0.9(0.1-6.1)$ & 0.95 & $0.6(0.1-6.9)$ & 0.70 & $0.7(0.1-6.5)$ & 0.73 & $0.5(0.0-2.3)$ & 0.41 \\
\hline \multicolumn{9}{|l|}{ Upper ESD } \\
\hline - none & 1 & & 1 & & 1 & & 1 & \\
\hline - $1-90$ & $0.5(0.1-3.2)$ & 0.51 & $0.4(0.0-4.0)$ & 0.47 & $0.8(0.1-4.9)$ & 0.82 & $1.2(0.2-8.3)$ & 0.82 \\
\hline - 91-180 & $0.8(0.2-3.0)$ & 0.74 & $1.1(0.3-4.4)$ & 0.94 & $0.3(0.0-1.5)$ & 0.13 & $1.3(0.3-5.6)$ & 0.73 \\
\hline - $181 \leq$ & $1.4(0.7-3.1)$ & 0.64 & $1.4(0.6-3.3)$ & 0.68 & $0.6(0.2-1.4)$ & 0.52 & $5.7(1.3-25.0)$ & 0.02 \\
\hline \multicolumn{9}{|c|}{ Upper GI treatment } \\
\hline - none & 1 & & 1 & & 1 & & 1 & \\
\hline - $1-30$ & $2.4(0.4-13.6)$ & 0.34 & $0.7(0.1-3.9)$ & 0.72 & $0.3(0.1-2.0)$ & 0.25 & $1.5(0.3-7.3)$ & 0.62 \\
\hline - $31-60$ & $6.1(0.9-39.4)$ & 0.06 & $1.2(0.5-2.9)$ & 0.86 & $0.6(0.1-3.9)$ & 0.60 & $0.9(0.3-2.4)$ & 0.91 \\
\hline - $61 \leq$ & $4.1(0.7-25.1)$ & 0.12 & $1.2(1.0-1.3)$ & 0.85 & $0.6(0.3-1.4)$ & 0.56 & $2.7(1.2-5.9)$ & 0.21 \\
\hline \multicolumn{9}{|l|}{ Lower ESD } \\
\hline - none & 1 & & 1 & & 1 & & 1 & \\
\hline - 1-90 & $0.7(0.1-3.9)$ & 0.68 & $0.4(0.1-1.3)$ & 0.45 & $0.7(0.1-6.7)$ & 0.73 & $4.9(1.1-22.0)$ & 0.04 \\
\hline - 91-180 & $1.3(0.3-5.0)$ & 0.72 & $2.0(0.9-4.4)$ & 0.36 & $5.0(1.2-20.2)$ & 0.04 & $2.2(1.0-4.8)$ & 0.30 \\
\hline - $181 \leq$ & $1.2(0.2-7.7)$ & 0.87 & $1.8(0.3-12.4)$ & 0.57 & $1.0(0.1-11.2)$ & 0.98 & $4.5(0.8-24.0)$ & 0.08 \\
\hline \multicolumn{9}{|c|}{ Lower GI treatment } \\
\hline - $0-75$ & 1 & & 1 & & 1 & & 1 & \\
\hline . $76-525$ & $0.7(0.2-2.7)$ & 0.64 & $0.6(0.1-2.9)$ & 0.54 & $0.7(0.1-3.6)$ & 0.63 & $1.1(0.3-4.5)$ & 0.89 \\
\hline - $526 \leq$ & $1.0(0.2-4.0)$ & 0.99 & $1.3(0.3-5.4)$ & 0.75 & $2.0(0.4-10.1)$ & 0.39 & $5.6(2.3-13.3)$ & 0.05 \\
\hline \multicolumn{9}{|l|}{ Enteroscopy } \\
\hline - none & 1 & & 1 & & 1 & & 1 & \\
\hline - $1-225$ & $0.8(0.2-3.1)$ & 0.71 & $1.0(0.4-2.2)$ & 0.99 & $2.6(1.2-5.8)$ & 0.24 & $2.9(0.7-11.6)$ & 0.13 \\
\hline - $226 \leq$ & $0.6(0.1-2.9)$ & 0.47 & $0.8(0.3-1.9)$ & 0.78 & $1.8(0.4-9.3)$ & 0.47 & $3.9(1.0-15.8)$ & 0.06 \\
\hline \multicolumn{9}{|l|}{ ERCP } \\
\hline - none & 1 & & 1 & & 1 & & 1 & \\
\hline - $1-120$ & $1.3(0.2-8.6)$ & 0.77 & - & - & - & - & $1.9(0.3-14.3)$ & 0.52 \\
\hline - $121-270$ & $3.1(0.9-11.0)$ & 0.08 & $2.1(0.5-7.9)$ & 0.28 & $0.9(0.2-3.5)$ & 0.87 & $2.6(0.6-11.8)$ & 0.22 \\
\hline - $271 \leq$ & $0.4(0.1-2.5)$ & 0.33 & $0.2(0.0-1.3)$ & 0.10 & $0.4(0.1-2.1)$ & 0.28 & $2.6(0.6-12.5)$ & 0.23 \\
\hline
\end{tabular}


- Table 3 (Continuation)

\begin{tabular}{|c|c|c|c|c|c|c|c|c|}
\hline \multirow{2}{*}{$\begin{array}{l}\text { Total time } \\
\text { engaged in } \\
\text { (min/month) }\end{array}$} & \multicolumn{8}{|c|}{ Pooled adjusted OR ( $95 \% \mathrm{CI})$} \\
\hline & Neck & $P$ & Right shoulder & $P$ & Left shoulder & $P$ & Low back & $P$ \\
\hline \multicolumn{9}{|l|}{ EUS } \\
\hline - none & 1 & & 1 & & 1 & & 1 & \\
\hline - 1-90 & $1.6(0.4-6.3)$ & 0.52 & $0.4(0.1-2.4)$ & 0.31 & $0.2(0.0-1.2)$ & 0.08 & $0.9(0.2-5.0)$ & 0.95 \\
\hline . 91-180 & $1.8(0.4-7.3)$ & 0.42 & $2.3(0.5-10.3)$ & 0.28 & $0.5(0.1-2.5)$ & 0.41 & $2.6(0.6-11.2)$ & 0.19 \\
\hline - $181 \leq$ & $0.7(0.1-4.8)$ & 0.75 & $0.9(0.1-6.1)$ & 0.91 & $0.2(0.0-0.5)$ & 0.13 & $1.9(0.4-9.0)$ & 0.43 \\
\hline
\end{tabular}

cedure, and could not reveal other risk factors associated with neck pain.

\section{Low back MSDs}

Notably, longer total time per month of upper ESD, lower ESD, and lower gastrointestinal treatment were the risk factors significantly associated with symptoms in the low back. These endoscopic procedures were originally longer in comparison with the conventional endoscopic examination. A previous study revealed that the frequency of $>30$ colonoscopies per week was associated with low back MSDs, indicating that sitting when possible is useful for the prevention of low back and foot injuries [5]. In another report, endoscopists performing upper endoscopy while sitting had a lower prevalence of severe MSDs [6]. In contrast, a recent European study claimed that the sitting posture for environment modifications was a non-significant factor for the prevention of MSDs [12].

The discrepancy could be attributed to a variety of postures of endoscopists during performing. Prolonged standing or sitting posture may be related to musculoskeletal injuries, especially low back injuries. A recent trend in Ergonomics field can be found in sit-stand workstations designed to improve the alertness and performance of workers, as well as reduce the development of MSDs [30]. Such workstations allow workers to change their posture as they wish. We hypothesize that sitstand endoscopic workstations may diminish the occurrence of some endoscopy-related MSDs. A sit-stand workstation equipped with a unique wearable chair was developed to prevent laparoscopy-related MSDs. This chair can be fitted and removed rapidly and easily [31]. Furthermore, we demonstrated the usage of the ergonomic wobble stool for endoscopists, endoscopy nurses, and assistants in the endoscopy unit. This stool allowed staff to rapidly change from a sitting position to a standing position [32]. In the current survey, however, almost all endoscopists selected the standing position during these procedures: upper ESD (95.5\%), lower ESD (90.4\%), and lower gastrointestinal treatment (95.2\%). Therefore, we could not reveal the effect of endoscopic postures that influence low back injuries. Prospective studies are warranted to compare the re- duction of endoscopy-related MSDs between the sitting position, standing position, and sit-stand workstation.

\section{Shoulder MSDs}

Longer total time per month of lower ESD was a significant risk factor for symptoms in the left shoulder. Appropriate adjustment of the bed height to achieve a neutral posture of the head, neck, back, shoulders, and elbows of endoscopists has been recommended [22,27-29]. However, endoscopists have to lift the specific control of the endoscope using the left shoulder during the lower ESD procedure [29]. One of the ergonomic solutions might be use of an endoscopic holder for diminishing shoulder injury [33].

\section{Association between other endoscopic procedures and MSDs}

Enteroscopy, endoscopic retrograde cholangiopancreatography (ERCP), and interventional EUS require the usage of lead aprons and long endoscopy times. These procedures may also involve awkward postures for prolonged periods of time if the fluoroscopic, endoscopic, and ultrasound monitor is placed excessively high or to the side of the endoscopist [34]. It is thought that these procedures are more likely to lead to MSDs than other procedures $[8,22,27]$. However, in the current study, the time of these procedures was not a significant factor associated with MSDs. This discrepancy in the data may be attributed to the small sample size. Hence, further large surveys are warranted to confirm these findings.

\section{Practical implication of endoscopy-related work-rest schedule}

In contrast to 20 years ago, most gastroenterologists spend $>40 \%$ of their time performing endoscopies [9, 22]. The ASGE survey showed a relationship between the time spent performing endoscopy and the prevalence of endoscopy-related MSDs (>40\%, $P=0.002$ ) [9]. In the current study, endoscopic work occupied $42 \%$ of daily work. We have to recognize the working hours that gastroenterologists are engaged in endoscopy and the interaction between the endoscopists, devices, and workstation to reduce the occurrence of overuse injuries based on 
ergonomics. According to the ASGE survey, only $10 \%$ of endoscopists took regular breaks [9]. Endoscopy is a physically challenging procedure. Rest breaks during endoscopy are essential to provide the muscles, tendons, and ligaments a chance to recover from the strain exerted by the endoscopic procedure. Endoscopists should take care of their bodies and recognize the importance of stretching and microbreaks to avoid injury [27, 29, 35].

Furthermore, the manager of the endoscopic unit has to effectively manage the total time spent in endoscopic procedures per month to minimize the risk of endoscopy-related MSDs, especially during ESD and lower gastrointestinal treatment (e. g., polypectomy). The ergonomic timeout can ensure proper positioning and height of the monitor and bed, such that the head, neck, back, shoulders, and elbows are in neutral postures $[11,22]$. Furthermore, the ergonomic timeout should emphasize the need for regular breaks during endoscopy before the initiation of longer endoscopic procedures.

Another practical implication of this study provides an important holistic view of endoscopic related MSDs. Work-related MSDs of medical workers have been analyzed with a focus on ergonomics $[3,26]$. Endoscopy-related MSDs in the endoscopic unit are not limited to endoscopists. Studies are also required to assess the MSDs occurring in endoscopic nurses, endoscopic technicians, and sanitation staff. Education in ergonomics for endoscopists and endoscopic staff is warranted to prevent the development of endoscopy-related MSDs later in their career [28].

\section{Study limitations}

Some limitations of this study have to be acknowledged. Although the response rate on the current survey was generally sufficient (51.6\%), the larger samples were needed to evaluate the role of recent diagnostic and therapeutic endoscopic procedures (e.g., enteroscopy, ERCP, and interventional EUS) in the development of MSDs. Odds ratios could not calculate due to the low number of endoscopy-related MSDs in the hands and wrists. Furthermore, recall bias may have influenced the responses of the participants. In our survey, the number of endoscopists who required environments modifications and specific treatments for endoscopy-related MSDs were not investigated.

\section{Conclusions}

This study revealed that most endoscopists struggle against endoscopy-related MSDs mainly on neck, shoulders, and low back. We also found evidence suggesting a direct correlation between the endoscopy-related procedure time of ESD or lower gastrointestinal treatment and the risk of MSDs mainly low back and left shoulder. Managing monthly total endoscopic time, in light of organizational ergonomics, could contribute to minimizing such risks of endoscopy-related MSDs.

\section{Acknowledgments}

The authors thank all of the endoscopists who participated in this study.

\section{Competing interests}

The authors declare that they have no conflict of interest.

References

[1] Hildebrandt VH, Bongers PM, van Dijk Fj et al. Dutch Musculoskeletal Questionnaire: description and basic qualities. Ergonomics 2001; 44: 1038-1055

[2] Smith AC, Wolf JG, Xie GY et al. Musculoskeletal pain in cardiac ultrasonographers: results of a random survey. J Am Soc Echocardiogr 1997; 10: 357-362

[3] van Det M], Meijerink W], Hoff C et al. Optimal ergonomics for laparoscopic surgery in minimally invasive surgery suites: a review and guidelines. Surg Endosc 2009; 23: 1279-1285

[4] Buschbacher R. Overuse syndromes among endoscopists. Endoscopy 1994; 26: 539-544

[5] Liberman AS, Shrier I, Gordon PH. Injuries sustained by colorectal surgeons performing colonoscopy. Surg Endosc 2005; 19: 16061609

[6] Byun YH, Lee JH, Park MK et al. Procedure-related musculoskeletal symptoms in gastrointestinal endoscopists in Korea. World J Gastroenterol 2008; 14: 4359-4364

[7] Hansel SL, Crowell MD, Pardi DS et al. Prevalence and impact of musculoskeletal injury among endoscopists: a controlled pilot study. J Clin Gastroenterol 2009; 43: 399-404

[8] Kuwabara T, Urabe Y, Hiyama T et al. Prevalence and impact of musculoskeletal pain in Japanese gastrointestinal endoscopists: a controlled study. World J Gastroenterol 2011; 17: 1488-1493

[9] Ridtitid W, Cote GA, Leung W et al. Prevalence and risk factors for musculoskeletal injuries related to endoscopy. Gastrointest Endosc 2015; 81: 294-302 e294

[10] Austin K, Schoenberger $\mathrm{H}$, Sesto $\mathrm{M}$ et al. Musculoskeletal Injuries Are Commonly Reported Among Gastroenterology Trainees: Results of a National Survey. Dig Dis Sci 2019; 64: 1439-1447

[11] Villa E, Attar B, Trick W et al. Endoscopy-related musculoskeletal injuries in gastroenterology fellows. Endosc Int Open 2019; 7: E808-E812

[12] Morais R, Vilas-Boas F, Pereira P et al. Prevalence, risk factors and global impact of musculoskeletal injuries among endoscopists: a nationwide European study. Endosc Int Open 2020; 8: E470-E480

[13] Shergill AK, Asundi KR, Barr A et al. Pinch force and forearm-muscle load during routine colonoscopy: a pilot study. Gastrointest Endosc 2009; 69: 142-146

[14] Campbell EV, Muniraj T, Aslanian H. Musculoskeletal pain syndromes and injuries among endoscopists who perform ERCP. Dig Dis Sci 2020: doi:10.1007/s10620-020-06163-z

[15] Kaewboonchoo O, Yamamoto H, Miyai N et al. The Standardized Nordic Questionnaire Applied to Workers Exposed to Hand-Arm Vibration. Journal of Occupational Health 1998; 40: 218-222

[16] Rubin D. Multiple imputation for nonresponse in surveys. NY: John Wiley \& Sons; 1987

[17] Rubin D. Multiple Imputation after $18+$ years. Journal of the American Statistical Association 1996; 91: 473-489 
[18] Ende AR, De Groen P, Balmadrid BL et al. Objective differences in colonoscopy technique between trainee and expert endoscopists using the colonoscopy force monitor. Dig Dis Sci 2018; 63: 46-52

[19] Anderson JT. Optimizing ergonomics during endoscopy training. Techniques in Gastrointestinal Endoscopy 2019; 21: 143-149

[20] Berguer R, Hreljac A. The relationship between hand size and difficulty using surgical instruments: a survey of 726 laparoscopic surgeons. Surg Endosc 2004; 18: 508-512

[21] Cohen DL, Naik JR, Tamariz LJ et al. The perception of gastroenterology fellows towards the relationship between hand size and endoscopic training. Dig Dis Sci 2008; 53: 1902-1909

[22] Shergill AK, McQuaid KR, Rempel D. Ergonomics and GI endoscopy. Gastrointest Endosc 2009; 70: 145-153

[23] Khanicheh A, Shergill AK. Endoscope design for the future. Techniques in Gastrointestinal Endoscopy 2019; 21: 167-173

[24] Shergill AK, Harris Adamson C. Failure of an engineered system: The gastrointestinal endoscope. Techniques in Gastrointestinal Endoscopy 2019; 21: 116-123

[25] Cromie JE, Robertson V], Best MO. Work-related musculoskeletal disorders in physical therapists: prevalence, severity, risks, and responses. Phys Ther 2000; 80: 336-351

[26] Matern U, Faist M, Kehl K et al. Monitor position in laparoscopic surgery. Surg Endosc 2005; 19: 436-440
[27] Pedrosa MC, Farraye FA. ASGE Technical Committee, . et al. Minimizing occupational hazards in endoscopy: personal protective equipment, radiation safety, and ergonomics. Gastrointest Endosc 2010; 72: $227-235$

[28] Harvin G. Review of musculoskeletal injuries and prevention in the endoscopy practitioner. J Clin Gastroenterol 2014; 48: 590-594

[29] Shergill AK, McQuaid KR. Ergonomic endoscopy: An oxymoron or realistic goal? Gastrointest Endosc 2019; 90: 966-970

[30] Ebara T, Kubo T, Inoue T et al. Effects of adjustable sit-stand VDT workstations on workers' musculoskeletal discomfort, alertness and performance. Ind Health 2008; 46: 497-505

[31] Matsuzaki I, Ebara T, Tsunemi M et al. Sit-stand endoscopic workstations equipped with a wearable chair. VideoGIE 2019; 4: 498-500

[32] Tsunemi M, Matsuzaki I, Hattori M et al. Sit-stand endoscopic workstations with wobble stools for the endoscopist, assistant, and endoscopy nurses in an endoscopy unit. Endoscopy 2020; 52: E324325

[33] Rattan J, Rozen P. A new colonoscope holder. Dis Colon Rectum 1987; 30: 639-640

[34] O'Sullivan S, Bridge G, Ponich T. Musculoskeletal injuries among ERCP endoscopists in Canada. Can J Gastroenterol 2002; 16: 369-374

[35] Siau K, Anderson JT. Ergonomics in endoscopy: Should the endoscopist be considered and trained like an athlete? Endosc Int Open 2019; 7: E813-E815 